\title{
Anatomía microscópica y metabolitos secundarios volátiles en tres estadios del desarrollo de las inflorescencias de Lantana camara (Verbenaceae)
}

\author{
José Fernando Caroprese Araque ${ }^{1}$, María Isabel Parra Garcés², Dagoberto Arrieta Prieto ${ }^{3}$ \\ \& Elena Stashenko ${ }^{4}$ \\ 1,2,3. Laboratorio de Histotecnia, Universidad Industrial de Santander, carrera 27 con Calle 9 Ciudad Universitaria UIS, \\ Bucaramanga, Colombia; teléfono (57+7)6344000 ext. 2354, fax 6349088; josfercar@ gmail.com¹, \\ marysapgar@gmail.com² ${ }^{2}$ darrieta@uis.edu.co ${ }^{3}$ \\ 4. Laboratorio de Cromatografía, CIBIMOL-CENIVAM, Universidad Industrial de Santander, carrera 27 con Calle 9 \\ Ciudad Universitaria UIS, Bucaramanga, Colombia; teléfono (57+7) 6344000 ext.2786, fax 6454104; \\ elena@tucan.uis.edu.co
}

\section{Recibido 25-VIII-2009. Corregido 10-VIII-2010. Aceptado 09-IX-2010.}

\begin{abstract}
Microscopic anatomy and volatile secondary metabolites at three stages of development of the inflorescences of Lantana camara (Verbenaceae). Plants of the Verbenaceae family, like L. camara, have called the attention of researchers, not only because of its high diversity and its distribution around the world, but also for its variable use as popular medicine to treat diseases like tetanus, rheumatism and malaria, and as bactericide and insecticide. To assess this, the morphology and ontogeny of the inflorescences of Lantana camara and the chemical composition of volatile secondary metabolites were analyzed at three different ontogeny stages. Plants were collected from the experimental crop area in CENIVAM, Bucaramanga, Colombia. Fresh inflorescence stages were established and analyzed using a stereoscopic microscope, fixed in FAA and included in parafine. Transversal and longitudinal $10 \mu \mathrm{m}$ thick sections were prepared using a rotative microtome, safranine-fastgreen stained and were observed and photographed using a light microscope. The chemical composition of volatile secondary metabolites were analyzed for each stage. The analytes, obtained from $0.7 \mathrm{~g}$ of plant, were isolated by solid phase micro-extraction in the headspace mode (HS-SPME) and were placed in $20 \mathrm{ml}$ vials. The components were analyzed by gas chromatography coupled to mass spectrometry (GC-MS). Stage I was microscopically characterized by an immature development in which the meristematic differentiation begins with a mass of cells. In Stage II, the morphogenetic movement gives way to the formation of the respective floral sexual structures, calyx and corolla. In Stage III, the different organs are conspicuous: four stamens epipetals and didynamous, monocarpelar, biloculate and globose gynoecium, upper ovary and lateral stigma; the flowers are hermaphroditic. The main secondary metabolites detected by GC-MS were bicyclosesquiphellandrene, E- $\beta$-farnesene, E- $\beta$-caryophyllene, $\gamma$-muurolene $+\gamma$-curcumene and $\alpha$-zingiberene. Nevertheless, this study reports for the first time in plant species $\alpha$-gurjunene, $\gamma$-amorphene, $\alpha$-muurolene, sesquithujene, $\alpha$-trans-bergamotene and transcadina-1,4-diene. The diversity of compounds found can be only explained by the extraction methods employed, the developmental stages and section of the plant, the geographic conditions, collection time and the genetic constitution of the evaluated species. Rev. Biol. Trop. 59 (1): 473-486. Epub 2011 March 01.
\end{abstract}

Key words: Lantana camara, Verbenaceae, inflorescences, floral morphology and anatomy, ontogeny, volatile secondary metabolites, fragance.

Las plantas de la familia Verbenaceae se encuentran ampliamente distribuidas por casi todo el planeta, siendo más abundantes en las regiones tropicales y subtropicales, en forma de arbustos, hierbas y árboles. Poseen una gran diversidad botánica y han sido ampliamente estudiadas por su variable uso (Ocampo \& Valverde 2000). La especie Lantana camara L. (Verbenaceae), pertenece a esta familia en la que se incluyen cerca de 98 géneros con aproximadamente 2.500 taxones específicos (Ocampo \& Valverde 2000). Según Gupta 
(1995), L. camara también se designa taxonómicamente como Lantana armata Saucher, Camara vulgaris Benth, o Lantana aculeata L. En Colombia se conoce popularmente como "Venturosa", "Gurupacha" y "Cariaquillo".

Estudios realizados en la especie han demostrado la presencia de varios terpenoides, esteroides y alcaloides (Ghisalberti 2000). El alcaloide lantamina, obtenido de la corteza de las ramas y de las raíces de L. camara, muestra una intensa actividad antipirética y antiespasmódica, propiedad comparable con la de la quinina (Ghisalberti 2000). Sus hojas son también muy utilizadas en la medicina popular para el tratamiento de enfermedades como el tétano, el reumatismo y la malaria. Es reportada por ser una importante fuente de fósforo y potasio al emplearse como abono, por poseer propiedades farmacológicas, incluyendo la bactericida, insecticida, nematicida, antimutagénica y su acción repelente contra insectos vectores de la malaria, entre otras (Seyoum et al. 2002, Basu et al. 2005). En contraste con estos hallazgos, algunos de los compuestos de esta planta muestran efectos tóxicos para diferentes especies animales (Carrillo \& Díaz de Ramírez 2006).

En este estudio se eligieron y describieron tres estadios de desarrollo de L. camara y se identificaron simultáneamente los metabolitos secundarios volátiles que hacen parte de cada uno de ellos. Por primera vez se reportan estos datos. Se espera aportar un mayor conocimiento sobre los diversos eventos y caracteres involucrados en el desarrollo de las inflorescencias de la familia Verbenaceae y en especial, para esta especie. Se busca aportar información diagnóstica para la diferenciación intergenérica e identificar y reportar compuestos químicos volátiles que cambian a medida que avanza el desarrollo.

Los estudios acerca de la secuencia de iniciación y posición de los distintos órganos florales en formación, son importantes en la investigación relacionada con los controles moleculares de la morfogénesis floral (Hufford \& Diggle 1997, Brukhin et al. 2003), e igualmente, desde el punto de vista sistemático, como lo indica Yang et al. (2002) en sus trabajos sobre la posición y las relaciones de parentesco de algunos géneros de Solanaceae, $\mathrm{y}$ aunque este trabajo no tiene implicaciones directas sobre ello (controles moleculares de la morfogénesis floral y sistemática), se espera contribuir a futuros estudios acerca de la filogenia en Verbenaceae. Finalmente, sobre la importancia de los estudios químicos de los organismos (ya sea plantas o animales), recordamos el postulado que dice: "la diversidad de la flora y fauna es un resultado directo de las interacciones químicas y co-adaptaciones de plantas, herbívoros y microorganismos" (Mann 1987).

\section{MATERIALES Y MÉTODOS}

Material vegetal: Se inició con la recolección de muestras florales de L. camara en el cultivo experimental del Centro Nacional de Investigaciones para la Agroindustrialización de Especies Vegetales Aromáticas y Medicinales Tropicales (CENIVAM), de la Universidad Industrial de Santander (UIS), Bucaramanga, Colombia, al medio día. Este horario se eligió teniendo en cuenta el estudio realizado con una Verbenaceae (Lippia alba) por Duarte et al (2007), en el que se indica el incremento de producción de metabolitos secundarios en el horario mencionado. La identificación taxonómica de este material se efectuó en el Herbario Nacional Colombiano (COL 480749).

El material vegetal se observó en el microscopio estereoscopio (Carlzeiss Jena) para la identificación de las etapas I, II y III del desarrollo de las inflorescencias, teniendo en cuenta cambios de tamaño $(2.5,4$ y desde 20 hasta $34 \mathrm{~mm}$ respectivamente), de la forma (botón de flores pubescente, botón de flores con brácteas diferenciadas e inflorescencias en su máximo desarrollo, respectivamente) y del desarrollo (cambios observados a nivel microscópico: masa de células en diferenciación, asomo de los verticilos y verticilos completamente desarrollados, respectivamente). Se describió la morfología de cada una de las estructuras florales visibles. Los estadios seleccionados se fijaron en FAA (formol, ácido acético, 
alcohol), embebidos en parafina y seccionados con micrótomo rotatorio en cortes longitudinales y transversales de $10 \mu \mathrm{m}$. Posteriormente se sometieron a tinción con safranina-fastgreen (Johansen 1940). Los principales eventos del desarrollo morfoanatómico floral fueron observados y fotografiados para su descripción, en microscopio óptico (Olympus, Japón). Para las inflorescencias se tomaron como base las descripciones de Moreno (1984).

Análisis químico: El análisis químico se realizó según la metodología propuesta por Stashenko et al. (2004). Para el proceso de microextracción en fase sólida de la fase vapor (headspace) (HS-SPME) del material vegetal, se usó una fibra de poli (dimetilsiloxano)/ divinilbenceno (PDMS/DVB, 65 $\mu \mathrm{m}$ ) obtenida de Supelco Inc. (Bellefonte, Pennsylvania, USA). Gases especiales para cromatografía fueron obtenidos de Aga-Fano S.A. (Bucaramanga, Colombia). Se muestreó la fase vapor a $22 \pm 1^{\circ} \mathrm{C}$, de $0.7 \mathrm{~g}$ de tejido vegetal, colocada en un frasco de $20 \mathrm{ml}$. El tiempo de pre-equilibrio del frasco, fue de $15 \mathrm{~min}$ y el tiempo de exposición de la fibra fue de $40 \mathrm{~min}$, basado en experimentos preliminares. Las sustancias extraídas fueron térmicamente desorbidas a $250^{\circ} \mathrm{C}$, durante $2 \mathrm{~min}$, en un puerto de inyección del cromatógrafo de gases, usando un liner especial para SPME de volumen reducido.

El análisis cromatográfico de las muestras se realizó en un GC Hewlett-Packard (HP) 5890A Series II, equipado con un inyector split/splitless $\left(250^{\circ} \mathrm{C}\right.$, relación split $\left.1: 10\right)$ y un detector de ionización en llama (FID) $\left(250^{\circ} \mathrm{C}\right)$, por triplicado para cada muestra (en total nueve muestras). Los datos cromatográficos fueron adquiridos y procesados con un programa HP Chem Station HP Rev. A.06.03. Para la separación de mezclas se usó una columna capilar apolar, HP-5 de 30m x $0.25 \mathrm{~mm}$, D.I. x $0.25 \mu \mathrm{m}, \mathrm{d}_{\mathrm{f}}$, con fase estacionaria de $5 \%$-fenilpoli(metilsiloxano). La temperatura del horno fue programada de $45^{\circ} \mathrm{C}(2 \mathrm{~min})$ hasta $150^{\circ} \mathrm{C}$ a razón de $5 \mathrm{~min}^{-1}$ para la columna apolar, HP-5; y de $60^{\circ} \mathrm{C}(5 \mathrm{~min})$ hasta $280^{\circ} \mathrm{C}$ a razón de $8^{\circ} \mathrm{C}$ $\mathrm{min}^{-1}$ para la columna polar INNOWAX. El gas de arrastre empleado fue helio (99.9995\%, Aga-Fano, Bucaramanga S.A,), con una presión de entrada en la cabeza de la columna de 15 psi y una velocidad lineal de $26 \mathrm{~cm} / \mathrm{s}$. El gas auxiliar fue nitrógeno (99.995\%; AgaFano, Bucaramanga S.A), con una velocidad de flujo de $30 \mathrm{ml} / \mathrm{min}$. Las velocidades de flujos de gases de combustión en el FID, el aire y el hidrógeno, fueron de 300 y $30 \mathrm{ml} / \mathrm{min}$, respectivamente. Los espectros de masas se obtuvieron por impacto de electrones con energía de $70 \mathrm{eV}$, en un CG Agilent Technologies 6890 Plus acoplado a un detector selectivo de masas HP MSD 5972, Cromatografía de gases acoplada a espectrometría de masas (GC-MS) y equipado con un puerto de inyección split/ splitless (1:10), un inyector automático Agilent 7863, un sistema de datos HP MS ChemStation (Versión 1.05), incluyendo las bases de datos NBS 75K, WILEY 138K y ADAMS. Se usó una columna capilar de sílice fundida, HP-5MS de $30 \mathrm{mx} 0.25 \mathrm{~mm}$, D.I., con la fase estacionaria de $5 \%$-fenil-poli(metilsiloxano) de $0.25 \mathrm{~mm}$ de grosor. El gas de arrastre fue helio (99.9995\%, Aga-Fano, Bucaramanga S.A.), con una velocidad lineal de $35 \mathrm{~cm} / \mathrm{s}$. Las temperaturas de la cámara de ionización y de la línea de transferencia se mantuvieron a 230 y $285^{\circ} \mathrm{C}$, respectivamente. Los espectros de masas y corrientes iónicas reconstruidas (TIC) se obtuvieron en un cuádruplo, por medio de barrido automático de frecuencia (full scan), a $4.75 \mathrm{scan} \mathrm{s}^{-1}$, en el ámbito de masas de $m / z$ 40-350. La homogeneidad de cada pico cromatográfico fue revisada, obteniendo fragmentogramas de masas, basados en iones característicos de cada compuesto. Para la identificación de los compuestos se usaron espectros de masas e índices de retención de componentes de Kovàts (Kovàts 1965) de componentes, que se compararon con los existentes en la literatura (Adams 1995, Joulian \& König 1998).

Los datos fueron recopilados como media \pm error estándar de la media (SEM). Posteriormente, para establecer las diferencias significativas en la variación de los porcentajes relativos de los metabolitos secundarios volátiles de L. camara en las tres etapas de 
desarrollo, se realizó un análisis de varianza (ANOVA) de un vía tipo modelo lineal general (MLG), verificando previamente los supuestos de normalidad (prueba de Kolmogorov-Simirnov) y homogeneidad de varianzas (prueba de Levene). Por la naturaleza de los datos, éstos fueron transformados usando la raíz del arcoseno. Adicionalmente se utilizó la prueba de Tukey $(\mathrm{p}<0.05)$ para comparar las medias entre las diferentes etapas de desarrollo de todos los metabolitos encontrados. Los análisis estadísticos fueron realizados usando el programa SPSS para Windows, versión 13.0.

\section{RESULTADOS}

Morfología y anatomía floral: L. camara presenta inflorescencias en cabezuelas glomerulares, las cuales se caracterizan por un desarrollo inicial a partir de meristemos axilares. Estos meristemos se modifican durante la ontogenia variando en su tamaño y estructura (hecho que se corrobora en el análisis al microscopio).

Fueron elegidos tres estadios de desarrollo. El Estadio I (E1, ca 2.5mm) se caracterizó por su apariencia globosa y compacta (Fig. 1A y B). Microscópicamente, en dicho estadio se aprecia un desarrollo inmaduro representado por grupos de células (yema inicial) (Fig. 1C) en los cuales se inicia la diferenciación meristemática (Fig. 1D). Se observan además, previas a la aparición de los órganos, brácteas (con pubescencia lasciniada y con tricomas glandulares), que permanecerán dando soporte y protección a la flor y luego al fruto (Fig. 1E y F).

El Estadio II (E2, ca. 4mm) presenta notorios cambios estructurales que dan paso a una cabezuela globosa de mayor tamaño (Fig. 2A y B). A nivel microscópico se diferencian cada vez más los verticilos florales, iniciando por el cáliz y la corola (Fig. 2C). En este estadio los sépalos están fusionados en la parte apical para la protección del meristemo floral en desarrollo (Fig. 2D). Se aprecian las estructuras que darán origen al gineceo y al androceo (Fig. 2D) y cómo la bráctea, el cáliz y la corola protegen estos órganos; esta última en inicios del Estadio III (Fig. 2E y F).

En el Estadio III (E3, desde $20 \mathrm{~mm}$ hasta $34 \mathrm{~mm}$ ) surge la corola tubular bilabiada de coloración externa rojiza e interna amarillenta, pentalobulada, con simetría hipocrateriforme irregular o zigomorfa, posterior al desarrollo de los cálices y exhibiendo un desarrollo similar a estos (Fig. 3A y B). El cáliz es de color verde pálido, dentado, de apariencia membranosa, tubular y pubescente, al igual que las brácteas (Fig. 3B). De manera casi simultánea se diferencian y crecen, soldados en el interior y sobre la corola en elongación (epipétalos), los estambres en número de cuatro, con inserción perígina, didínamos, que en su origen se observan a la misma altura y tienen un color amarillo pálido, anteras ovadas ditecas y paralelas (Fig. 3B y E). El gineceo se diferencia de forma ascendente, formando así el ovario, el estilo y el estigma. El ovario es monocarpelar, súpero, ovoide, con dos lóculos, cada uno con un óvulo de placentación axial-axial. El estilo es glabro y el estigma es terminal lateral, diviso bilobulado de aspecto pegajoso que indica receptividad (Fig. 3B, C y D). De este modo, L. camara exhibe una maduración centrífuga (Fig. 3A y F). Así mismo presenta una estivación imbricada quincuncial, donde los pétalos uno y dos (los más externos), cubren de forma inmediata a los pétalos tres y cuatro (los pétalos fusionados). Estos a su vez, se encuentran inmediatamente cubriendo al pétalo cinco, el cual es el más interno (Fig. 3A). Microscópicamente, se observan las estructuras reproductoras de la planta. Al finalizar su periodo receptor, cada una de estas estructuras reproductoras se marchita y se originan los frutos drupáceos globosos que aún conservan las brácteas.

En cuanto a los resultados químicos, al igual que en la investigación realizada por Stashenko et al. (2004) en plantas de la familia Verbenaceae, para el caso de la especie L. camara, casi la totalidad de compuestos obtenidos en este estudio fueron del tipo sesquiterpenoide (90\%).

En el Cuadro 1 aparece la identificación de los metabolitos secundarios volátiles de flores 

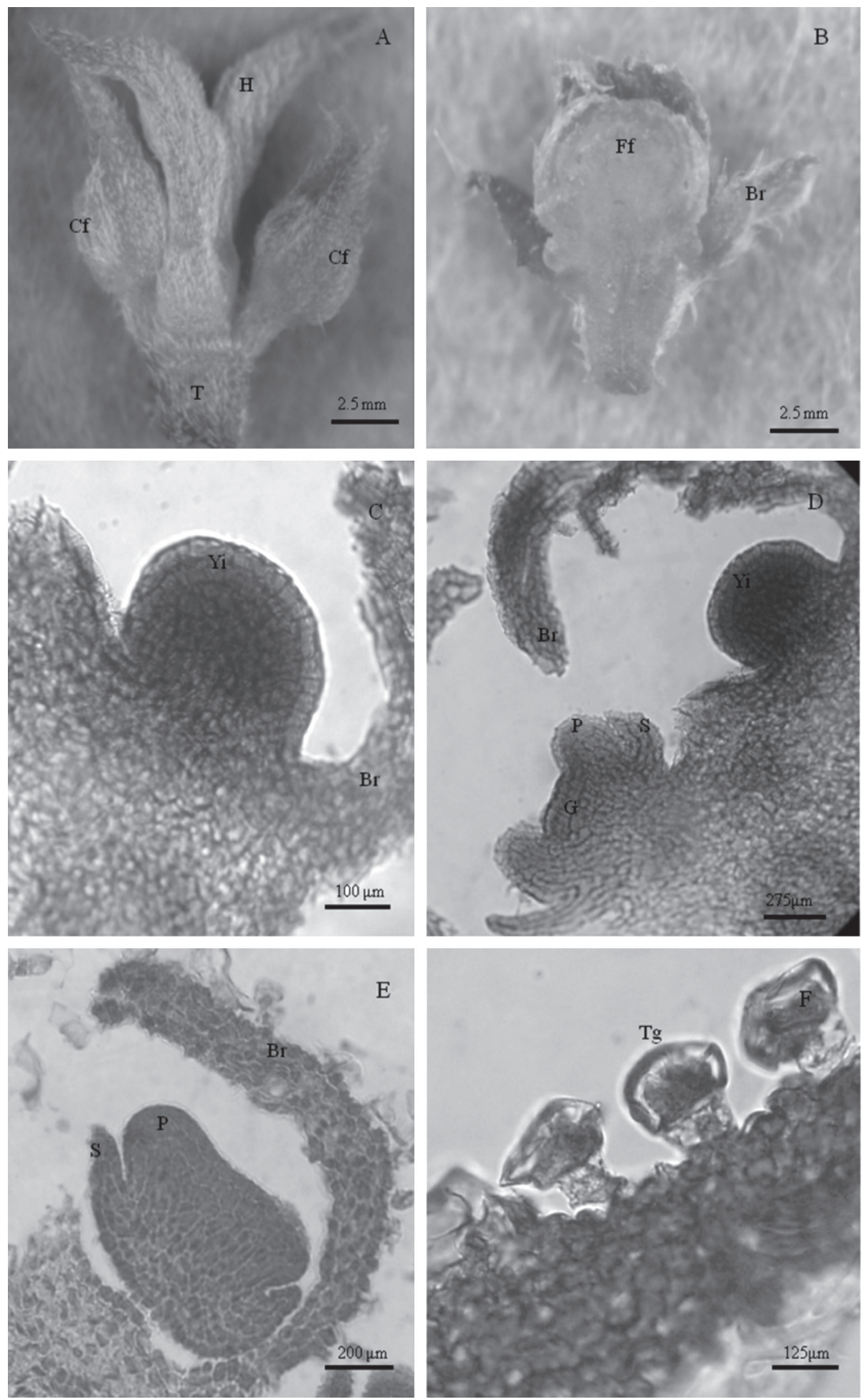

Fig. 1. Botón de flores (A y B) y cortes longitudinales (C, D, E, F) característicos del Estadio I. Abreviaturas: Yi: yema inicial; Br: bráctea; P: pétalos; S: sépalos; G: gineceo; Tg: tricoma glandular; H: hojas; T: tallo; Cf: cabezuela floral; Ff: flores en formación.

Fig. 1. Floral buds (A y B) and longitudinals cuts (C, D, E, F) particular of Stage I. Abbreviations: Yi: initial bud; Br: Bract; P: petals; S: sepals; G: gineceum; Tg: glandular trichome; H: leaves; T: branch; Cf: floral head; Ff: flowers in formation. 

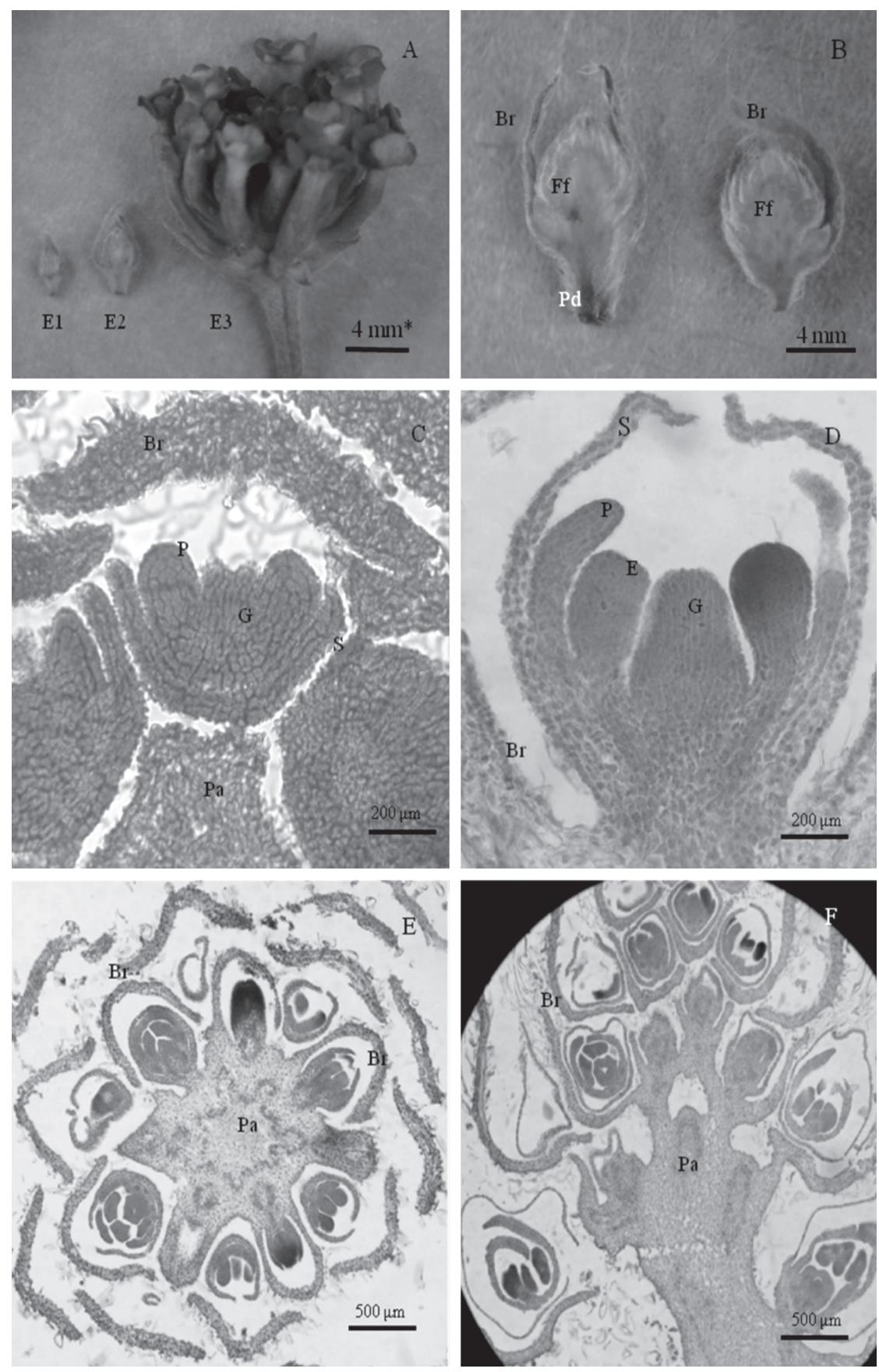

Fig. 2. Botones florales (A y B), cortes longitudinales (C, D, F) y transversal (E) característicos del Estadio II. Abreviaturas: Ff: flores en formación; Br: brácteas; Pd: pedicelo; P: pétalos; S: sépalos; E: estambres; G: gineceo; Pa: parénquima; E1: Estadio I; E2: Estadio II; E3: Estadio III. (*) Tamaño del Estadio II.

Fig. 2. Floral buds (A y B), longitudinals (C, D, F) and cross-sectional cuts (E) particular of Stage II. Abbreviations: Ff: flowers in formation; Br: bracts; Pd: pedicel; P: petals; S: sepals; E: stamens; G:gineceum; Pa: parenchime; E1: Stage I; E2: Stage II; E3: Stage III. (*) Size of Stage II. 

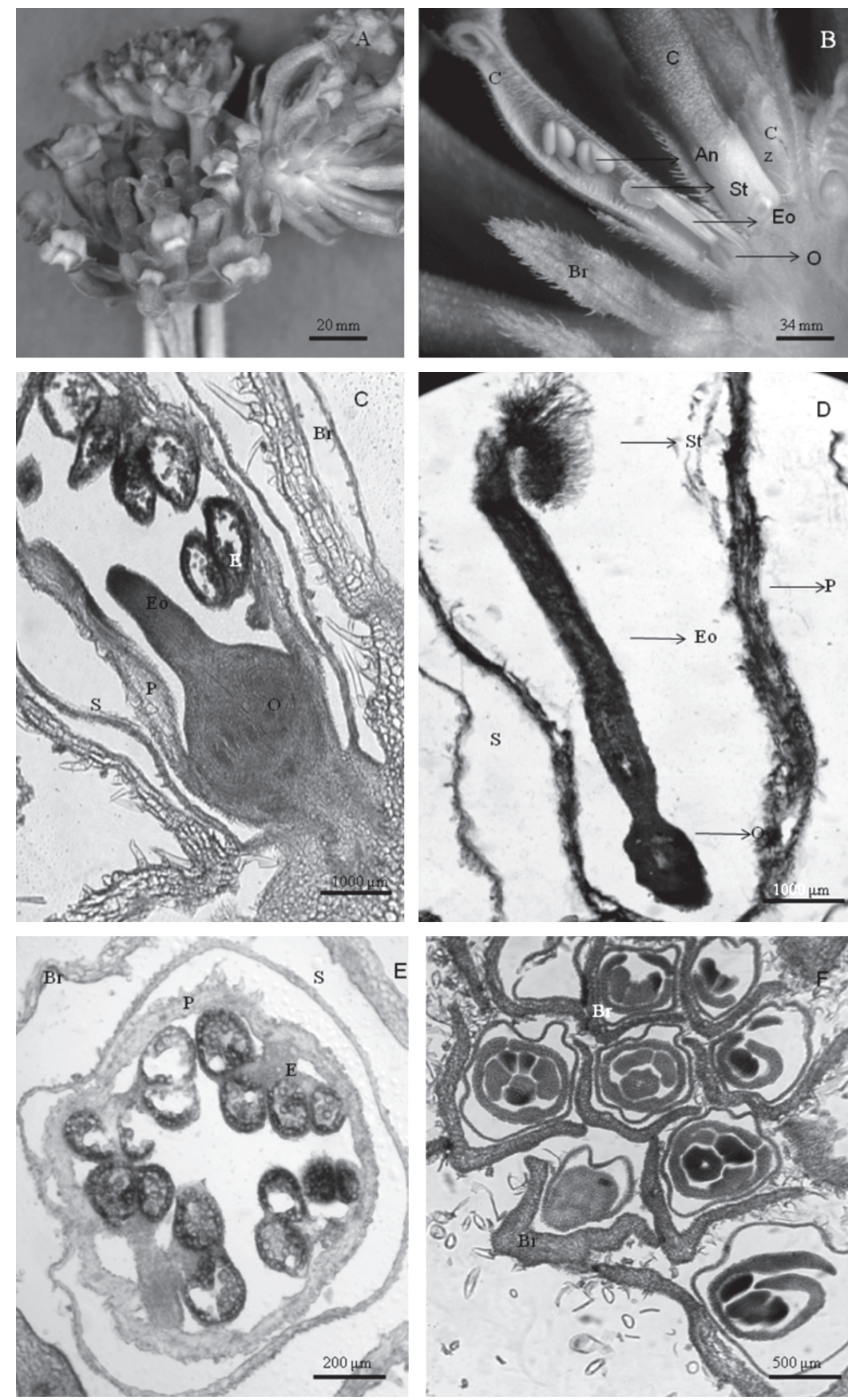

Fig. 3. Inflorescencias en su máximo desarrollo (A, B), cortes longitudinales (C, D, F) y transversal (E) correspondientes al Estadio III. Abreviaturas: Br: brácteas; C: corola; An: antera; St: estigma; Eo: estilo; O: ovario; Cz: cáliz; P: pétalos; E: estambres; S: sépalos.

Fig. 3. Inflorescences in their maximum development (A, B) and longitudinals (C, D, F) and cross-sectional cuts (E) belonging to Stage III. Abbreviations: Br: bracts; C: corolla; An: anthers; St: stigma; Eo: style; O: ovary; Cz: calyx; P: petals; E: stamens; S: sepals. 


\section{CUADRO 1}

Promedios $\pm S D$ de cantidad relativa de metabolitos volátiles en L. camara durante tres etapas de desarrollo ontegénico, número de pico e índices de Kovats

TABLE 1

Averages $\pm S D$ of essential oils in L. camara during three stages of ontogenic development, number of peaks and Kovats index

\begin{tabular}{|c|c|c|c|c|c|c|}
\hline \multirow{2}{*}{$\begin{array}{l}\text { N. }{ }^{\circ} \\
\text { pico }\end{array}$} & \multirow{2}{*}{$\begin{array}{l}\text { Indice } \\
\text { Kovats }\end{array}$} & \multirow{2}{*}{ Compuesto } & \multicolumn{4}{|c|}{ Cantidad relativa (\%) } \\
\hline & & & Estadio I & Estadio II & Estadio III & $\mathrm{P}$ \\
\hline 1 & 1031 & 1,8-Cineol & ND & $1.20 \pm 0.03$ & ND & - \\
\hline 2 & 1340 & $\delta$-Elemeno & $0.70 \pm 0.04 \mathrm{a}$ & $1.30 \pm 0.09 \mathrm{~b}$ & $0.90 \pm 0.07 \mathrm{a}$ & $\mathrm{p}<0.001^{* *}$ \\
\hline 3 & 1352 & $\alpha$-Cubebeno & $1.00 \pm 0.06 \mathrm{a}$ & $4.20 \pm 0.10 \mathrm{~b}$ & $3.50 \pm 0.11 \mathrm{c}$ & $\mathrm{p}<0.001 * *$ \\
\hline 4 & 1379 & $\alpha$-Copaeno & $0.70 \pm 0.02 \mathrm{a}$ & $1.30 \pm 0.09 \mathrm{~b}$ & $0.70 \pm 0.05 \mathrm{a}$ & $\mathrm{p}<0.01 *$ \\
\hline 5 & 1388 & $\beta$-Bourboneno & $0.30 \pm 0.01$ & ND & ND & - \\
\hline 6 & 1393 & $\beta$-Cubebeno ${ }^{\star}+\beta$-Elemeno & $1.10 \pm 0.03 \mathrm{a}$ & $2.40 \pm 0.05 \mathrm{~b}$ & $3.70 \pm 0.05 \mathrm{c}$ & $\mathrm{p}<0.001 * *$ \\
\hline 7 & 1408 & Sesquitujeno & ND & $0.70 \pm 0.03 \mathrm{a}$ & $1.40 \pm 0.08 \mathrm{~b}$ & $\mathrm{p}<0.01 *$ \\
\hline 8 & 1413 & $\alpha$-Gurjuneno & $0.30 \pm 0.02$ & ND & ND & - \\
\hline 9 & 1417 & $\alpha$-Cedreno & ND & ND & $0.80 \pm 0.11$ & - \\
\hline 10 & 1427 & $E$ - $\beta$-Cariofileno & $17.00 \pm 0.12 \mathrm{a}$ & $9.20 \pm 0.11 \mathrm{~b}$ & $13.00 \pm 0.08 \mathrm{c}$ & $\mathrm{p}<0.001 * *$ \\
\hline 11 & 1433 & $\beta$-Copaeno & $1.10 \pm 0.07 \mathrm{a}$ & $3.30 \pm 0.10 \mathrm{~b}$ & $4.90 \pm 0.09 \mathrm{c}$ & $\mathrm{p}<0.001 * *$ \\
\hline 12 & 1437 & $\alpha$-E-Bergamoteno & ND & $1.40 \pm 0.11$ & ND & - \\
\hline 13 & 1437 & $\gamma$-Elemeno & $1.70 \pm 0.04$ & ND & ND & - \\
\hline 14 & 1452 & $\alpha$-Humuleno & ND & $2.00 \pm 0.10 \mathrm{a}$ & $2.30 \pm 0.09 \mathrm{~b}$ & $\mathrm{p}<0.01^{*}$ \\
\hline 15 & 1463 & $E$ - $\beta$-Farneseno & $39.40 \pm 0.40$ & ND & ND & - \\
\hline 16 & 1465 & allo-Aromadendreno & ND & $2.20 \pm 0.07 \mathrm{a}$ & $1.50 \pm 0.06 \mathrm{~b}$ & $\mathrm{p}<0.01 *$ \\
\hline 17 & 1483 & $\gamma$-Muuroleno+ $\gamma$-Curcumeno & ND & $10.50 \pm 0.09 \mathrm{a}$ & $14.40 \pm 0.0 \mathrm{~b}$ & $\mathrm{p}<0.001 * *$ \\
\hline 18 & 1487 & Biciclosesquifelandreno & $17.60 \pm 0.04 \mathrm{a}$ & $31.10 \pm 0.04 \mathrm{~b}$ & $24.20 \pm 0.07 \mathrm{c}$ & $\mathrm{p}<0.001 * *$ \\
\hline 19 & 1499 & $\alpha$-Zingibereno & ND & $9.30 \pm 0.04 \mathrm{a}$ & $13.10 \pm 0.07 \mathrm{~b}$ & $\mathrm{p}<0.001 * *$ \\
\hline 20 & 1500 & $\gamma$-Amorfeno & $1.50 \pm 0.02$ & ND & ND & - \\
\hline 21 & 1503 & NI & $0.80 \pm 0.04$ & ND & ND & - \\
\hline 22 & 1504 & $\alpha$-Muuroleno & $1.70 \pm 0.09 \mathrm{a}$ & $3.20 \pm 0.11 \mathrm{~b}$ & $3.00 \pm 0.09 \mathrm{c}$ & $\mathrm{p}<0.001 * *$ \\
\hline 23 & 1505 & $E, E$ - $\alpha$-Farneseno & $3.30 \pm 0.07 \mathrm{a}$ & $2.20 \pm 0.10 \mathrm{~b}$ & ND & $\mathrm{p}<0.001^{* *}$ \\
\hline 24 & 1513 & $\beta$-Curcumeno & ND & $2.50 \pm 0.08 \mathrm{a}$ & $4.00 \pm 0.05 \mathrm{~b}$ & $\mathrm{p}<0.01^{*}$ \\
\hline 25 & 1517 & $\gamma$-Cadineno & $2.60 \pm 0.04 \mathrm{a}$ & $2.50 \pm 0.07 \mathrm{a}$ & $1.60 \pm 0.06 \mathrm{~b}$ & $\mathrm{p}<0.001 * *$ \\
\hline 26 & 1525 & $\delta$-Cadineno & $4.60 \pm 0.08 \mathrm{a}$ & $5.10 \pm 0.09 \mathrm{~b}$ & $3.00 \pm 0.07 \mathrm{c}$ & $\mathrm{p}<0.001 * *$ \\
\hline 27 & 1534 & E-Cadina-1,4-dieno & ND & $0.70 \pm 0.08 \mathrm{a}$ & $0.60 \pm 0.06 \mathrm{~b}$ & $\mathrm{p}<0.001^{* *}$ \\
\hline 29 & 1539 & $\alpha$-Cadineno & $1.10 \pm 0.04 \mathrm{a}$ & $0.50 \pm 0.02 \mathrm{~b}$ & $0.20 \pm 0.01 \mathrm{c}$ & $\mathrm{p}<0.01^{*}$ \\
\hline 30 & 1559 & Germacreno B & $1.20 \pm 0.08 \mathrm{a}$ & $0.50 \pm 0.05 \mathrm{~b}$ & $0.70 \pm 0.09 \mathrm{c}$ & $\mathrm{p}<0.001 * *$ \\
\hline
\end{tabular}

** Probabilidades altamente significativas

* Probabilidades significativas

Identificado tentativamente

$\mathrm{ND}=$ No Detectado

$\mathrm{P}=$ Probabilidad

Significancia según test de Anova, letras diferentes (a, b, c) indican diferencias significativas, según el test de Tukey $(\mathrm{p}<0.05)$ 
de L. camara, aislados mediante HS-SPME con sus cantidades relativas $(\% \pm \mathrm{SD}, \mathrm{n}=3)$. En el Estadio I se detectaron 20 compuestos y se identificaron 19; en el Estadio II, 22 fueron detectados y 20 en el Estadio III, todos en cantidades relativas $>0.05 \%$.

Durante la ontogenia de la inflorescencia, algunos de los metabolitos no se detectan en ciertos estadios. Así, se puede mencionar el $\beta$-bourboneno detectado únicamente en el Estadio I, el 1,8-cineol detectado sólo en el II y el $\alpha$-cedreno, que sólo fue detectado en el Estadio III. Esta es una condición presente en varios de los compuestos, al igual que las variaciones en sus cantidades relativas.

\section{DISCUSIÓN}

Los eventos ontogénicos ocurren para todas las especies, siendo importante saber dónde y cuándo se activan ciertos genes, pero también las dinámicas autoorganizadas que se generan a partir de las interacciones de éstos (Álvarez \& Roces 2002). Como parte de la expresión fenotípica (tamaño, forma y desarrollo de verticilos) se establecieron tres estadios, el mismo número elegido por Garzón \& Orozco (2006) para su estudio. El Estadio I finaliza, al verse la elongación y reacomodamiento celular que da paso a la aparición de los incipientes cáliz y corola. Así mismo, L. camara exhibe una maduración centrípeta de los verticilos (brácteas, sépalos, pétalos, estambres y gineceo) y de cada una de las coloridas flores hermafroditas que conforman la inflorescencia, es decir, se aprecian flores ya abiertas en la parte distal de densos glomérulos (más de 25 flores), mientras en la parte central de la inflorescencia aún se presentan botones florales. Uno de los hechos representativos de ello es la necesidad de los pétalos para el surgimiento de los estambres debido a la posición epipétala de éstos. El desarrollo de las inflorescencias a lo largo de las ramas de esta especie vegetal se da de manera acrópeta, observándose la disposición en filotaxia espiral al eje, de modo que las inflorescencias más desarrolladas se encuentran en la parte basal y en la apical se aprecian aún en botón.

A diferencia de los cuatro pétalos reportados por Muñoz et al. (2007) para Lippia alba, L. camara presenta cinco pétalos, dos de ellos fusionados dando la impresión de ser uno solo. Sin embargo, el aspecto zigomorfo, la bráctea que soporta a cada una de las flores que conforman la inflorescencia, el ovario súpero y los cuatro estambres epipétalos y didínamos se mantienen como característicos en las dos especies. La línea de ruptura para la liberación de los granos de polen se observó a lo largo de la antera, siendo de este modo, una dehiscencia longitudinal. Es notorio el aspecto receptivo que presenta el estigma, antes y durante esta apertura, caracterizado por la producción de una sustancia mucilaginosa. Este fenómeno es reportado en otras especies vegetales como Carica papaya (Ronse \& Smets 1999, Parés et al. 2002).

Las características de la organogénesis floral de L. camara corresponden a un patrón presente en la mayoría de las angiospermas. Los verticilos emergen siguiendo la regla de Hofmeister (los nuevos órganos se originan hacia la parte central en los espacios inmediatamente disponibles), al igual que lo reportado por Garzón \& Orozco (2006) en su investigación con Solanáceas.

Múlgura de Romero et al. (2002) describen la exomorfología de las inflorescencias en Verbenáceas, mencionando el carácter sésil de las flores, excepto en algunas especies de Aloysia, Xerolaoysia, Stachytarpheta sp. y Chascanum sp., donde se presentan cortos pedicelos. De igual modo, la escasa presencia de bracteolas florales que sólo se presenta en Bouchea y en Chascanum. Estas características generales se acogen a lo observado en este estudio para L. camara.

Los cambios que pueden presentarse entre las diferentes especies de Verbenáceas en cuanto a la disposición, espaciamiento y número de flores de las inflorescencias, son muy notorios a pesar de su cercanía (Múlgura de Romero et al. 2002). Así, L. camara y L. alba presentan caracteres compartidos en los eventos de 
su desarrollo floral, tales como la secuencia centrípeta en el surgimiento de los verticilos florales y la disposición que ellos adquieren, pero mantienen diferencias en cuanto al tipo de inflorescencia y sus frutos (drupáceos y secos respectivamente). Así mismo, L. camara y Duranta erecta se diferencian en que esta última presenta un cáliz con cinco sépalos, cuatro carpelos, estigma capitado y bractéolas, en contraste con las características ya mencionadas de la especie bajo estudio (Múlgura de Romero et al. 2002).

Una de las estructuras que siempre se encuentra presente en todos los estadios de desarrollo sobre las brácteas, los cálices y las corolas, son los tricomas (Fig. 1F). Éstos, al parecer, están relacionados con la producción de metabolitos secundarios (aceites y volátiles) que atraen y repelen insectos y otorgan a la planta las diferentes propiedades que, por supuesto, tienen notorias implicaciones en el ambiente y sus organismos. Tal es el caso mencionado por Mann (1987), acerca de los metabolitos cardenolidos, presentes en las hojas de algunas plantas y cómo su consumo por parte de las larvas de la mariposa monarca las hacen desagradables a un extenso rango de depredadores. El mismo autor dice que algunos de los aleloquímicos son generados por activación fotoquímica y por la degradación de los compuestos originales producidos por la planta, que son luego liberados al ambiente. Esta característica que habla de la condición lumínica, fue tomada en cuenta, al igual que otros estudios hechos con Verbenáceas (Duarte et al. 2007), para la elección del medio día como horario de muestreo floral y su inmediato análisis químico.

Al contrastar los metabolitos encontrados en la presente investigación con los de otros estudios, igualmente de L. cámara, se observan diferencias significativas. Stashenko et al. (2004) reporta más de 35 metabolitos secundarios volátiles aislados del aceite esencial extraído de la mezcla de hojas y flores con E- $\square$-cariofileno (15\%) y biciclosesquifelandreno $(18 \%)$ como mayoritarios. Por su parte Adeleke et al. (2004) encontraron 39 compuestos en inflorescencias, con sabineno (21.5\%) y 1,8-cineol (14.8\%) en mayor cantidad. Virendra et al. (2005) reportan 30 compuestos en hojas, con $\beta$-cariofileno (23.3\%) y $\alpha$-humuleno $(11.5 \%)$ como principales. Randrianalijaona et al. (2005) reportan 50 compuestos en el aceite extraído de inflorescencias con sabineno (14.3\%) y 1,8-cineol (1.0\%) como mayoritarios. Khan et al. (2003) obtuvieron más de 30 compuestos en flores, con el $\alpha$-copaeno ( $10.7 \%)$ y el $\beta$-elemeno ( $14.5 \%$ ) como componentes mayoritarios. En este estudio, se identificaron 29 metabolitos (Cuadro 1), mediante HS-SPME. No obstante, esta diferencia en la diversidad de compuestos se puede ver justificada al tener en cuenta los métodos de extracción empleados (Stashenko et al. 2004), las partes de la planta con sus estadios de desarrollo sometidas a ellos, al igual que las condiciones geográficas, época de recolección de la muestra vegetal y constitución genética (Nickavar \& Mojab 2000). El $\alpha$-gurjuneno, el $\gamma$-amorfeno, el $\alpha$-muuroleno, el sesquitujeno, el $\alpha$-trans-bergamoteno y el trans-cadina1,4-dieno son, respecto a las investigaciones previamente citadas, compuestos hallados en L. camara que se reportan por vez primera en el presente estudio.

Al estudiar los compuestos volátiles correspondientes a cada uno de los estadios seleccionados, se verificaron las variaciones en la cantidad relativa de éstos a medida que avanza el proceso de desarrollo floral, así como la presencia y ausencia de algunos de ellos (Cuadro 1). Mann (1987) menciona que esta fluctuación está muy relacionada con el desarrollo del organismo, su estatus nutricional y los niveles de estrés ambiental (depredadores y adaptación, incluyendo cambios en los genes), entre otros. Bouwmeester et al. (1998) reportaron la síntesis de carvona a expensas del limoneno en frutos de Carum carvi (alcaravea). En el presente estudio, al parecer ocurre un proceso similar (Cuadro 1), ya que sólo en el Estadio I, fueron detectados compuestos como el trans$\beta$-farneseno (39.4\%), el $\gamma$-elemeno (1.7\%) y el $\gamma$-amorfeno (1.5\%), mientras el $\alpha$-zingibereno (13\%), el $\gamma$-muuroleno+ $\gamma$-curcumeno (14\%) y 
el $\beta$-curcumeno (4\%), entre otros, sólo en los estadios II y III. Acerca de la producción de metabolitos secundarios, Dudareva et al (1996) reportan, por ejemplo, que la expresión del gen responsable de la síntesis de metilbenzoato, en petunia, es suprimida por el etileno. Igualmente, Hallahan et al. (1995) y Bouwmeester et al. (1998) mencionan cómo los terpenos alcohólicos geraniol y carveol son convertidos en aldehídos por una deshidrogenasa no específica, mientras el geranial y el neral son coproducidos por la oxidación del geraniol. No obstante, para la presente investigación con $L$. camara, estas transformaciones solo podrían confirmarse mediante un análisis químico como marcaje isotópico y el conocimiento de sus rutas metabólicas.

Es probable que para L. camara la expresión de los diversos genes en los verticilos florales, origine diferentes compuestos y en las contrastantes cantidades observadas (Cuadro 1), como ha sido comprobado por Dudareva et al (1996) en flores de Clarkia breweri. Estos órganos, en esta planta, emiten una fuerte fragancia a causa de su compuesto mayoritario: el s-linalool, un monoterpeno. Para ello, el gen Lis está altamente expresado en las células del estigma, del estilo y de las células epidérmicas de los pétalos, al igual que en los estambres. No obstante, en Clarkia concinna el gen Lis sólo es expresado en el estigma y a un nivel relativamente bajo.

Para la presente investigación, el $\beta$-bourboneno, el $\gamma$-elemeno y el $\gamma$-amorfeno, se detectaron sólo en el Estadio I donde se aprecian las yemas en diferenciación, mientras el monoterpeno oxigenado 1.8-cineol se detectó junto con el $\alpha$-trans-bergamoteno, sólo en el Estadio II, estadio en el cual las divisiones celulares y el cumplimiento del plan morfoanatómico permiten la estimación visual de lo que será cada uno de los órganos florales. Otros metabolitos como el trans- $\beta$-cariofileno y el biciclosesquifelandreno estuvieron presentes en las tres etapas, es decir, hasta la presencia de los verticilos florales maduros, tomando en cuenta que para esta investigación, fue tomada la inflorescencia en su conjunto. Para verificar los metabolitos y las cantidades producidas de forma individual, hubiese sido necesario el análisis de cada uno de los órganos por separado.

Acerca de la producción y emisión de compuestos volátiles, Dudareva et al. (2000) y Gershenzon et al. (2000) mencionan que estas son reguladas por procesos del desarrollo. Cuando las flores jóvenes aún no han hecho la dehiscencia, producen menos esencias y por ello, son menos atractivas a los polinizadores. Lo anterior se encontró de forma similar para esta investigación; así, L. camara presenta la mayor variedad de compuestos volátiles durante el segundo y tercer Estadios (botón de flores) de desarrollo (Cuadro 1). Algunas de las cantidades relativas de los metabolitos, fueron aumentando a medida que avanzó la ontogenia (Cuadro 1). Es probable entonces que permanecieran para este estadio de apertura floral, los compuestos necesarios cualitativamente y cuantitativamente para diversas funciones, entre ellas, la atracción de los polinizadores, reflejando así las preferencias olfatorias de éstos y haciendo que la planta produzca distintas fragancias, tal como cita Dudareva et al.(1996). Igualmente persistirían y se incrementarían los metabolitos adecuados para proteger a las flores en su antesis y dehiscencia (Dudareva \& Pichersky 2006). En relación con lo anterior, estudios realizados con las flores polinizadas de Antirrhimum majus y Petunia sp., verificaron que se detiene la producción de esencias sólo después de que el polen ha encontrado el ovario, seguramente cuando la fertilización ha ocurrido (Dudareva et al. 1996). Por otra parte, el metilbenzoato, que es uno de los compuestos más abundantes encontrado en las flores de Antirrhimum majus, tiene regulada su emisión durante el desarrollo (Dudareva et al. 2000). Así mismo, algunos de los compuestos que en el Estadio III no son detectados en L. camara (Cuadro $1)$, tales como el trans- $\beta$-farneseno y el transtrans- $\alpha$-farneseno, dejarían de ser sintetizados durante algún acontecimiento ocurrido a través del proceso ontogénico. Algo similar ocurrió también en estudios realizados por Tollsten 
(1993) con Platanthera bifolia (Orchidaceae) y por Schiestl et al. (1997) y Schiestl \& Ayasse (2001) con la orquídea Ophrys, en la cual, los cambios en la emisión de las sustancias volátiles luego de la polinización, incluyeron una disminución general de todos los componentes de las esencias o una reducción en algunos de los compuestos mientras otros se incrementaron o permanecieron sin cambiar.

Varios estudios se han realizado para reportar las diversas funciones que se presumen para los metabolitos secundarios (incluidos los volátiles). Una de estas funciones es aumentar la tolerancia de la fotosíntesis a las temperaturas altas, estabilizando las membranas tilacoidales (Sharkey et al. 2001) o controlando las especies con oxígeno reactivo (Loreto \& Velikova 2001). Se ha discutido también la posibilidad de que estas substancias actúen en la comunicación planta-planta (Arimura et al. 2000, Dicke \& Bruin 2001, Engelberth et al. 2004), además de minimizar el daño extenso de los tejidos (Dicke \& Van Loon 2000) y actuar como repelentes de los herbívoros y patógenos (Andersen et al. 1994, De Moraes et al. 2001, Kessler \& Baldwin 2001). Estos volátiles inducidos por los herbívoros, podrían tener adicionalmente papeles fisiológicos dentro de la planta, al ser descargados como consecuencia de su volatilidad y solubilidad desde la membrana. Compuestos como el isopreno, algunos monoterpenos y sesquiterpenos, tienen el potencial para combinarse con varias especies de oxígeno reactivo (radicales libres), protegiendo contra el daño interior oxidativo (Hoffmann et al. 1997, Bonn \& Moortgat 2003). Según sugiere Dudareva et al (2004), las próximas investigaciones requieren el estudio de la importancia de los volátiles para la fisiología de la planta.

\section{AGRADECIMIENTOS}

Los autores expresan sus más sinceros agradecimientos a María Nilda Cerf Arbulú y a José Miguel Esborronda Andrade, por la contribución con sus conocimientos y sugerencias para la realización de este proyecto. A la Federación Internacional de Sociedades Científicas
(FISS) por el material bibliográfico. Agradecemos igualmente a Jhon Jairo Díaz Olarte por su apoyo en el diseño y revisión estadística, a Nestor Marín por sus asesorías en la parte química, a Humberto García Pinzón por sus aportes en la descripción de las inflorescencias, a Edgar Rincón Barón y a José Caroprese Quintero, por contribuir con su experiencia a esta investigación.

\section{RESUMEN}

Las plantas de la familia Verbenaceae, entre ellas la especie Lantana camara L., poseen una alta diversidad botánica además de su variable uso. Se describe el avance morfoanatómico de tres etapas de desarrollo de las inflorescencias de L. camara L. (Verbenaceae), recolectadas en Bucaramanga, Colombia. Para esto se tincionaron las estructuras vegetales mediante la técnica fast-green, previo a los análisis con microscopía óptica convencional. El aislamiento de los analitos de cada estadio se realizó mediante la técnica de microextracción en fase sólida en el modo headspace (HS-SPME) y su posterior análisis de componentes, mediante cromatografía de gases acoplada a espectrometría de masas (GC-MS). En el Estadio I se apreció un grupo de células en donde se inicia la diferenciación meristemática. En el Estadio II, los movimientos morfogenéticos dan paso a la formación de las respectivas estructuras sexuales florales, el cáliz y la corola. Al llegar al Estadio III, se observaron claramente los diferentes órganos. Los metabolitos detectados en este estudio y que no fueron encontrados en la bibliografía revisada fueron, el $\alpha$-gurjuneno, el $\gamma$-amorfeno, el $\alpha$-muuroleno, el sesquitujeno, el $\alpha$-trans-bergamoteno y el trans-cadina-1,4-dieno. Los principales metabolitos secundarios detectados fueron: biciclosesquifelandreno, E- $\beta$-farneseno, E- $\beta$-cariofileno, $\gamma$-muuroleno+ $\gamma$-curcumeno y $\alpha$-zingibereno. Una función importante de éstas y otras esencias florales es la defensa y la protección durante el estrés abiótico de la planta.

Palabras clave: Lantana camara, Verbenaceae, morfología y anatomía floral, ontogenia, metabolitos secundarios volátiles, fragancia.

\section{REFERENCIAS}

Adams, R.P. 1995. Identification of essential oil components by gas chromatography/mass spectrometry. Allured, Carol Stream, Illinois, EEUU.

Adeleke, A., E. Olusegun, W. Koenig \& P. Yadua. 2004. Essential Oil of Lantana camara $L$. var. aculeata from Nigeria. J. of Essent. 16: 582-584. 
Álvarez, E. \& B. Roces. 2002. La diversidad de las formas vegetales. Variaciones sobre un mismo tema. Ciencias 65: 18-28.

Andersen, R.A., T.R. Hamilton, D.F. Hildebrand, C.T. Jr. McCracken, R.W. Collins \& P.D. Fleming. 1994. Structure-antifungal activity relationships among volatile $\mathrm{C}_{6}$ and $\mathrm{C}_{9}$ aliphatic aldehydes, ketones and alcohols. J. Agric. Food. Chem. 42: 1563-1568.

Arimura, G., R. Ozawa, T. Shomoda, T. Nishioka, W. Boland \& J. Takabayashi. 2000. Herbivory-induced volatiles elicit defense genes in lima bean leaves. Nature 406: 512-515.

Basu, S., A. Ghosh \& B. Hazra. 2005. Evaluation of the antibacterial activity of Ventilago madraspatana Gaertn., Rubia cordifolia Linn. and Lantana camara Linn.: Isolation of emodin and physcion as active antibacterial agents. Phytother. Res.19: 888-894.

Bonn, B. \& G.K. Moortgat. 2003. Sesquiterpene ozonolysis: origin of atmospheric new particle formation from biogenic hydrocarbons. Geophys. Res. Lett. 30: $1585-1593$

Bouwmeester, H.J., J. Gershenzon, M. Konings \& R. Croteau. 1998. Biosynthesis of the monoterpenes Limonene and Carvone in the Fruit of Caraway. Demonstration of enzyme activities and their changes with development. Plant Physiol. 117: 901-912.

Brukhin, V., H. Michel, N. González, C. Chevalier \& A. Mouras. 2003. Flower development schedule in tomato Lycopersicon esculentum cv. sweet cherry. Sex Plant Reprod. 15: 311-320.

Carrillo, R. \& A. Díaz de Ramírez. 2006. Actividad antimalárica de extractos acuosos de Lantana camara L., Verbena littoralis L. y Heliotropium indicum L. en ratones infectados con Plasmodium berghei. Rev. Fac. Farma. 48: 14-20.

De Moraes, C., M. Mescheer \& J. Tumlinson. 2001. Caterpillar-induced nocturnal plant volatiles repel nonspecific females. Nature 410: 577-580.

Dicke, M. \& J. Bruin. 2001. Chemical information transfer between plans: back to the future. Biochem. Syst. Ecol. 29: 981-994.

Dicke, M. \& J. Van Loon. 2000. Multitrophic effects of herbivore-induced plant volatiles in an evolutionary context. Entomol. Exp. Appl. 97: 237-249.

Duarte, S., C. Cárdenas, J. Martínez \& E. Stashenko. 2007. Estudio de la variación circadiana de los metabolitos secundarios volátiles obtenidos por la destilación extracción con solvente simultánea de hojas de Lippia alba (fam. Verbenaceae). Scient Et Tech. 13: 83-85.

Dudareva, N. \& E. Pichersky. 2006. Biology of floral Scent. Taylor \& Francis, Nueva York, EEUU.

Dudareva, N., E. Pichersky \& J. Gershenzon. 2004. Biochemistry of Plant Volatiles. Plant Physiol. 135: 1893-1902.

Dudareva, N., L. Murfitt, C. Mann, N. Gorenstein, N. Kolosova, C. Kish, C. Bonham \& K. Wood. 2000. Developmental regulation of methylbenzoate biosynthesis and emission in snapdragon flowers. Plant Cell 12: 949-961.

Dudareva, N., L. Cseke, V. Blanc \& E. Pichersky. 1996. Evolution of floral scent in Clarkia: novel patterns of $S$-linalool synthase gene expression in the $C$. breweri flower. Plant Cell 8: 1137-1148.

Engelberth, J., H. Alborn, E. Schmelz \& J. Tumlinson. 2004. Airborne signals prime plants against insect herbivore attack. Proc. Natl. Acad. Sci. USA 101: 1781-1785

Garzón, J. \& C. Orozco. 2006. Organogénesis floral en Acnistus arborescens, Dunalia solanacea, Deprea bitteriana, Larnax glabra and Larnax hawkesii- tribu Physaleae (Solanaceae). Caldasia 28: 227-242.

Gershenzon, J., M. McConkey \& R. Croteau. 2000. Regulation of monoterpene accumulation in leaves of peppermint. Plant Physiol. 122: 205-213.

Ghisalberti, E. 2000. Review Lantana camara L.Verbenaceae. Fitoterapia71: 467-486.

Gupta, M. 1995. 270 plantas medicinales iberoamericanas. CYTED-SECAB, Bogotá, Colombia.

Hallahan, D., J. West, R. Wallsgrove, D. Smiley, G. Dawson, J. Pickett \& J. Hamilton. 1995. Purification and characterization of an acyclic monoterpene primary alcohol: $\mathrm{NADP}^{+}$oxidoreductase from catmint (Nepeta racemosa). Arch. Biochem. Biophys. 318: 105-112.

Hoffmann, T., J. Odum, F. Bowman, D. Collins, D. Klockow, R. Flagan \& J. Seinfeld. 1997. Formation of organic aerosols from the oxidation of biogenic hydrocarbons. J. Atmos. Chem. 26: 189-212.

Hufford, L. \& P. Diggle. 1997. The morphology and evolution of flowers: a tribute to the work of Shirley Tucker: an introduction. Int. J. Plant. Sci. 158: 1-2.

Johansen, D. 1940. Plant microtechnique. McGraw-Hill, Nueva York, EEUU. 
Joulain, D. \& W. König. 1998. The atlas of spectral data of sesquiterpene hydrocarbons. EB-Verlag, Hamburgo, Alemania.

Kessler, A. \& I. Baldwin. 2001. Defensive function of herbivore-induced plant volatile emissions in nature. Science 291: 2141-2144.

Khan, S., K. Srivastava, J. Neetu, K. Syamasundar \& K. Anju. 2003. Chemical composition of fruit and stem essential oils of Lantana camara from northern India. Flavour Fragance J. 18: 376-379.

Kovats, E. 1965. Gas chromatographic characterization of organic substances in the retention index system. Adv. Chromatogr. 1: 229-247.

Loreto, F. \& V. Velikova. 2001. Isoprene produced by leaves protects the photosynthetic apparatus against ozone damage, quenches ozone products, and reduces lipid peroxidation of cellular membranes. Plant Physiol. 127: 1781-1787.

Mann, J. 1987. Secondary Metabolism. Oxford Science, Nueva York, EEUU.

Moreno, N. 1984. Glosario Botánico Ilustrado. Compañía Editorial Continental, México.

Múlgura de Romero, M., S. Martínez, S. Atkins \& A. Rotman. 2002. Morfología de las inflorescencias en Verbenáceas, Verbenoideae III. Darwiniana 40: 1-15.

Muñoz, A., F. Vallejo \& M. Sánchez. 2007. Morfología y anatomía de las flores y semillas de pronto alivio. Ac. Agron. Col. 56: 7-11.

Nickavar, B. \& F. Mojab. 2000. Hydrodistilled volatile constituents of Calamintha officinalis Moench from Iran. J. Essent. Oil-Bear. Plants 8: 23-27.

Ocampo, R. \& R. Valverde. 2000. Manual de cultivo y conservación de plantas medicinales. Tomo I. Instituto de Investigaciones Fundamentales en Agricultura Tropical, Costa Rica.

Parés, J., C. Basso \& D. Jáuregui. 2002. Momento de antesis, dehiscencia de anteras y receptividad estigmática en flores de lechosa (Carica papaya L.) cv. Cartagena amarilla. Bioagro. 14: 17-24.

Randrianalijaona, J., A. Panja, R. Rasoarahona \& E. Gaydou. 2005. Seasonal and chemotype influences on the chemical composition of Lantana camara L. Essential oils from Madagascar. Anal. Chim. Acta 545: 46-52.

Ronse, L. \& E. Smets. 1999. The floral development and anatomy of Carica papaya (Caricaceae). Can. J. Bot. 77: 582-598.

Schiestl, F. \& M. Ayasse. 2001. Post-pollination production of a repellent compound in a sexually deceptive orchid: a new mechanism for maximising reproductive success? Oecologia 126: 531-534.

Schiestl, F., M. Ayasse, H. Paulus, D. Erdmann \& W. Francke. 1997. Variation of floral scent emission and post pollination changes in individual flowers of Ophrys sphegodes subsp. sphegodes (Miller). J. Chem. Ecol. 23: 2881-2895.

Seyoum, A., E. Kabiru, W. Lwande, G. Killeen, A. Hassanali \& B. Knols. 2002. Repellency of live potted plants against Anopheles gambiae from human baits in semi-field experimental huts. Am. J. Trop. Med. Hyg. 67: 191-195.

Sharkey, T.D., X.Y. Chen \& S. Yeh. 2001. Isoprene increases thermotolerance of fosmidomycin-fed leaves. Plant Physiol. 125: 2001-2006.

SPSS para Windows. 2004. Versión 13.0. Chicago: SPSS Inc. [programa informático en CD-ROM]. (También disponible en línea: http://www.spss.com/).

Stashenko, E., B. Jaramillo \& J. Martínez. 2004. Comparación de la composición química y de la actividad antioxidante in vitro de los metabolitos secundarios volátiles de plantas de la familia Verbenaceae. Rev. Acad. Col. Cienc. Exact. Fis. Nat. 27: 579-59

Tollsten, L. 1993. A multivariate approach to post-pollination changes in the floral scent of Platanthera bifolia (Orchidaceae). Nord. J. Bot. 13:495-499.

Virendra, S., D. Prasad \& A. Blazquez. 2005. Chemical Composition of the Leaf Oil of Lantana camara. J. Essent. Oil Res. 17: 198-200.

Yang, D., Z. Zhang, A. Lu, K. Sun \& J. Liu. 2002. Floral organogénesis and development of two taxa in the tribe Hyoscyameae (Solanaceae) Przewalskia tangutica and Hyoscyamus niger. Acta Bot. Sin. 44: 889-894. 